\title{
CRANIOSTENOSE EM GÊMEOS: ESTUDO GENÉTICO
}

\author{
Walter Carlos Pereira * \\ Nélio Garcia de Barros ** \\ Gilberto Machado de Almeida * \\ Pedro Henrique Saldanha ***
}

A etiologia da craniostenose permanece obscura até o presente. Virchow (cit. por Ingraham e Matson ${ }^{8}$ ), em 1851, sugeriu que ela seria causada por meningites ou osteomielites; desde então a craniostenose tem sido atribuída a sífilis, riquetzioses, traumatismos pré-natais ou durante o parto, disfunções endócrinas e infecções intra-uterinas ${ }^{5}, 7,8$. Nenhuma dessas etiologias foi, contudo, suficientemente comprovada. Estudos reiterados dos ossos envolvidos nunca demonstraram anormalidades significativas, quer macro, quer microscòpicamente ${ }^{7,8}$. Reilly ${ }^{9}$ admite que alguns casos de craniostenose possam estar relacionados com o raquitismo. Alguns autores atribuem a obliteração precoce das suturas do crânio a defeito fundamental do plasma germinativo $6,7,8$.

A transmissão hereditária é indiscutível em certas formas de craniostenose, tais como a disostose craniofacial (moléstia de Crouzon) e a acrocefalossindactilia (síndrome de Apert) ${ }^{1}$. Nestas afecções geralmente há vários individuos afetados na mesma família, há grande predominância de acometimento de crianças do sexo masculino e associação freqüente com outras anomalias do desenvolvimento embrionário. Esse conjunto de deformidades, provocadas por inibição ou retardo do desenvolvimento do embrião, parece ser transmitido hereditàriamente por gens autossômicos dominantes ${ }^{4,10}$. No entanto, têm sido descritos casos esporádicos da síndrome de Apert, atribuídos a fenômeno de neomutação por Blank (cit. por Degenhardt ${ }^{4}$ ).

Em relação à obliteração precoce da sutura coronária ("oxicefalia") e da sutura sagital ("escafocelafia") não há estudos minuciosos que demonstrem claramente o fator hereditário causal. Ésses tipos de craniostenose apresentam-se, em geral, desacompanhados de malformaçōes de outros órgãos. A oxicefalia quase sempre ocorre de maneira esporádica, sendo raros os casos familiares relatados ${ }^{4,10}$. A escafocefalia, em alguns casos, apresenta transmissão hereditária do tipo dominante irregular ${ }^{4}$. Contudo, a maioria dos trabalhos a êste respeito carece de dados genealógicos pormenorizados ou se basea em número reduzido de casos ${ }^{2,3}$.

Dos Departamentos de Neurologia e de Genética da Faculdade de Medicina da Universidade de São Paulo: * Neurocirurgião; ** Neurorradiologista; *** Professor de Genética. 
O problema etiológico da craniostenose tem sido encarado de maneira secundária pela maioria dos autores que se dedicaram ao estudo dessa afecção. Por êsse motivo, julgamos útil o relato que ora fazemos da ocorrência de duas formas clínicas diversas de craniostenose, em gêmeos de sexo diferente, com a respectiva análise genética.

\section{OBSERVAČ́ES}

I. F. C., sexo feminino (registro 458.571) e E. F. C., sexo masculino (registro 458.570), gêmeos com 9 anos de idade, de côr branca. Foram encaminhados ao ambulatório da Clínica Neurológica por apresentarem deformidade craniana e deficiência mental, notada principalmente após iniciarem as atividades escolares. Antecedentes - Os pais são primos em segundo grau. A mãe, por ocasiāo do parto, tinha 20 anos de idade e o pai, 24. A gestação foi aparentemente normal, não havendo rerefência a moléstias infecciosas, traumatismos, uso de drogas ou radioterapia. O parto ocorreu prematuramente, por volta do oitavo mês de gravidez, sendo necessário o uso de fórcipe em virtude de provável inércia uterina. Ambas as crianças nasceram com sinais de hipoxia; após serem submetidas a manobras de reanimação foram mantidas em incubadora durante um mês. $O$ desenvolvimento psicomotor foi retardado nos dois pacientes: sentaram sem apoio por volta de um ano; mantiveram-se em pé aos dois anos; começaram a falar as primeiras palavras aos três anos e sòmente desenvolveram razoàvelmente a linguagem aos quatro anos de idade. Sempre se mostraram crianças extremamente irritáveis e irriquietas. Freqüentam escola há mais de dois anos e apenas aprenderam e escrever as vogais. Exame clinico-neurológico - Ambas as crianças apresentam estado geral satisfatório, estando o desenvolvimento somático ligeiramente atrazado em relação à idade cronológica. O exame dos diferentes aparelhos e sistemas nada revela digno de destaque, a não ser no que se refere ao segmento cefálico. O exame neurológico, nos dois pacientes, também se mostra dentro dos limites da normalidade.

Exame do segmento cefálico - Caso I.F.C.: crânio achatado no sentido anteroposterior, com diâmetro vertical aumentado; perímetro craniano $43,5 \mathrm{~cm}$; distância anteroposterior $26 \mathrm{~cm}$; distância biauricular $27,5 \mathrm{~cm}$; discreto exoftalmo bilateral simétrico; som levemente timpânico à percussão do crânio. Caso E.F.C.: crånio alongado anteroposteriormente e bastante estreito; região frontal ampla e projetada para frente; perímetro craniano $51,5 \mathrm{~cm}$; distância anteroposterior $36 \mathrm{~cm}$; distância biauricular $31 \mathrm{~cm}$; assimetria facial discreta, com predomínio da hemiface esquerda sôbre a direita; som de "pote rachado" à percussão do crânio.

Exames complementares - Caso I. F. C. - Craniogramas: aumento acentuado das impressões digitiformes; obliteração das suturas coronária e porção anterior da sagital (fig. 1A). Electrencefalograma normal. Testes psicológicos (Goodenough e Terman-Merril): QI 60. Caso E.F.C. - Craniogramas: aumento das impressōes digitiformes e obliteração da sutura sagital (fig. 1B). Electrencefalograma normal. Testes psicológicos (Goodenough e Terman-Merril): QI 62.

Análise genética - $\mathrm{O}$ exame cariotípico de um dos gêmeos (E.F.C.) indica que a craniostenose é independente de aberracōes cromossômicas. A análise cromossômica foi feita mediante cultura temporária de leucócitos, segundo técnica modificada de Moorhead. Examinaram-se onze metáfases, tôdas exibindo 46 cromossomos, incluindo 5 pares do grupo $G$ e 15 pares do grupo $C$, como seria de se 
esperar em se tratando de individuo do sexo masculino. A figura 2 mostra o aspecto metafásico de uma das células examinadas, assim como o pareamento dos cromossomos classificados de acôrdo com a nomenclatura de Denver. O cariótjpo dêste individuo evidencia número e configuração normais dos cromossomos. Éste resultado está de acôrdo com o padrão de herança sugerido pela análise genealógica (fig. 3). Os pacientes são filhos de pais consangüineos, são gêmeos dizigóticos (sexos diferentes) e foram os únicos afetados em tôda a genealogia. Estes fatos indicam que a craniostenose é determinada por gens recessivos raros de natureza autossômica e afastam a possibilidade de fenocópias ou interferências de fatôres ambientais.

Evolução - Ambos os doentes foram tratados cirùrgicamente. A paciente I. F. C. foi operada em 6-9-1966, sendo verificadas, durante o ato cirúrgico, obliteraçāo total da sutura coronária e quase total da sutura sagital, que se encontrava aberta apenas na sua porção mais posterior. Foram efetuadas craniectomias lineares bilaterais paralelas às referidas suturas. O paciente E. F. C. foi operado em 31-5-1966, sendo encontrada obliteração exclusiva da sutura sagital em tôda sua extensão. Visando a finalidades estéticas, além de duas craniectomias lineares paralelas à sutura obliterada, foram realizadas craniotomias osteoplásticas nas regiões temporais para possibilitar o aumento do diâmetro transversal do crânio.

Nenhuma complicação foi registrada em conseqüência do ato cirúrgico. Após a alta hospitalar os pacientes continuaram a ser observados no ambulatório até março de 1967, quando a familia mudou-se para outra cidade, nunca mais voltando para consulta. Até aquela ocasião não foi observada modificação apreciável no comportamento dos pacientes, assim como também não houve variação nas dimensões cranianás dos mesmos. Infelizmente, não foi possivel realizar novos testes para avaliar a evolução pós-operatória do psiquismo dos doentes.

\section{COMENTÁRIOS}

Os fatôres hereditários determinantes da obliteração precoce da sutura sagital e da sutura coronária não estão ainda bem estudados, havendo poucos relatos na literatura sôbre o assunto. Embora, no caso da escafocefalia, sejam conhecidas famílias com vários indivíduos afetados 2, 6, 10, são também registrados com freqüência casos esporádicos dessa anomalia ${ }^{4,10}$. Degenhardt ${ }^{4}$ sugere que a transmissão hereditária da escafocefalia seja do tipo dominante irregular. Por outro lado, a oxicefalia aparece quase sempre de maneira esporádica, sugerindo transmissão por gens recessivos. A concomitância de casos de escafocefalia e oxicefalia na mesma família é excepcionalmente descrita ${ }^{3}$.

O casal de gêmeos, que tivemos o ensêjo de estudar, exibia clìnicametne formas diferentes de craniostenose. A menina (I.F.C.) apresentava crânio alto e achatado no sentido anteroposterior e o menino (E.F.C.), crânio estreito no sentido transversal e alongado anteroposteriormente. Quanto a êste aspecto nossa observaçāo parece ser original, porquanto não encontramos referência alguma a caso semelhante. O exame radiológico revelou, em ambos, obliteração da sutura sagital, se bem que parcial na menina; a sutura coronária apresentava-se obliterada exclusivamente nesta (fig. $1 \mathrm{~A} \mathrm{e} \mathrm{B}$ ).

É óbvio que a análise genética da craniostenose só poderá ser considerada definitiva quando se basear no estudo de muitas familias com indiví- 

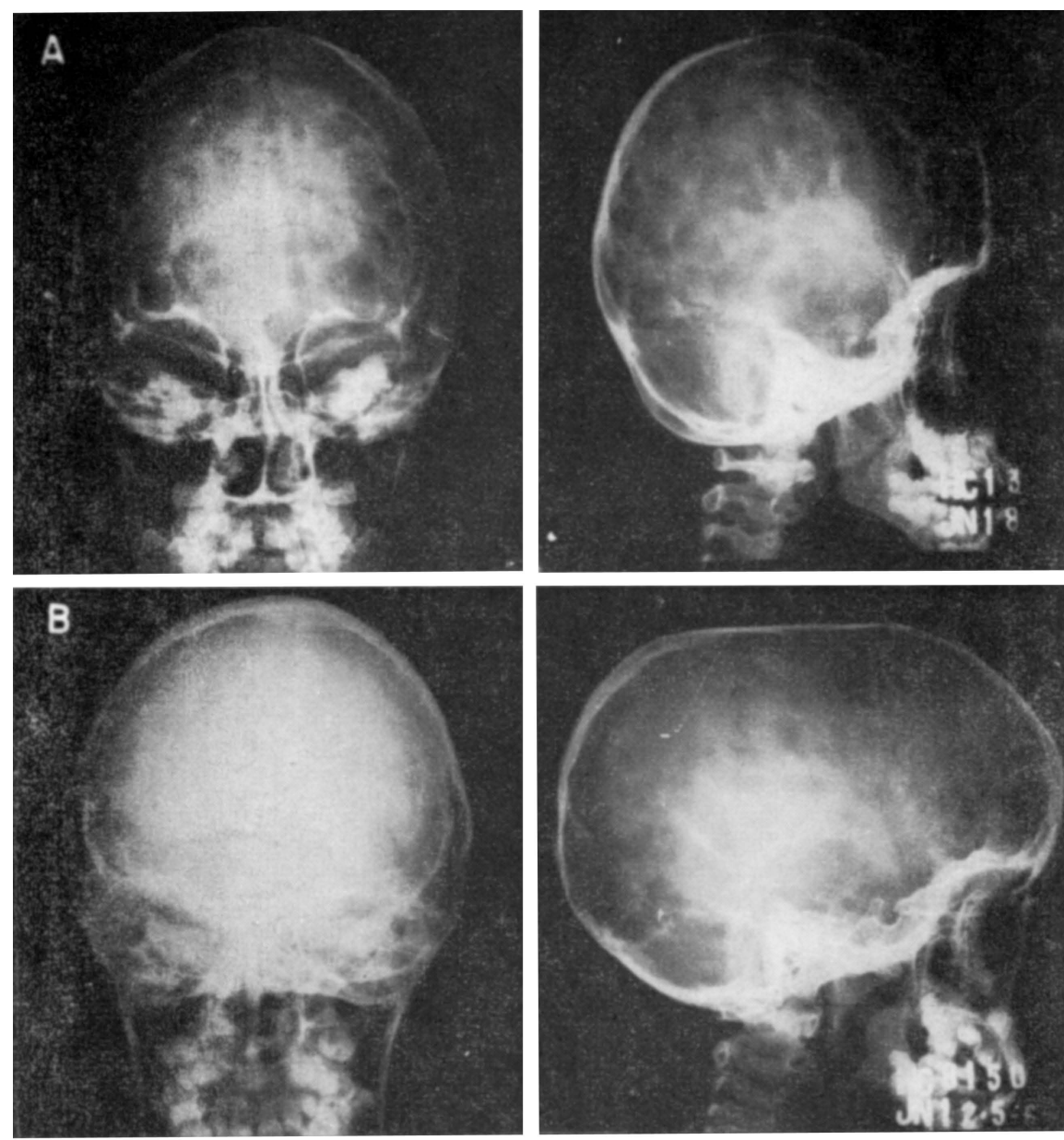

Fig. 1 - Craniogramas. Em A (caso I.F.C.), observa-se acentuado aumento das impressões digitiformes, assim como obliteração total da sutura coronária e parcial da sutura sagital. Em B (caso E.F.C.), nota-se acentuação das impressões digitiformes e obliteteraç̃o da sutura sagital.

duos afetados. Não obstante, o exame genealógico de nossos pacientes oferece feições características sôbre a etiologia genética dessa afecção. Os gêmeos estudados são filhos de pais consangüíneos, sendo os únicos afetados em tôda a genealogia. A consangüinidade é representada por primos em segundo grau com coeficiente de consagüinidade (probabilidade de homozigose entre os filhos) correspondente a $1 / 32$. O estudo da segregação de filhos normais e anômalos na familia está prejudicado pelo tamanho reduzido da prole, pois sòmente existe mais um filho com 6 anos de idade, que é normal. Todavia as demais características da genealogia sugerem que os pacientes com craniostenose são homozigotos para gens recessivos raros. A ocorrência de anômalos de ambos os sexos indica que êsses gens são de natureza autossô- 

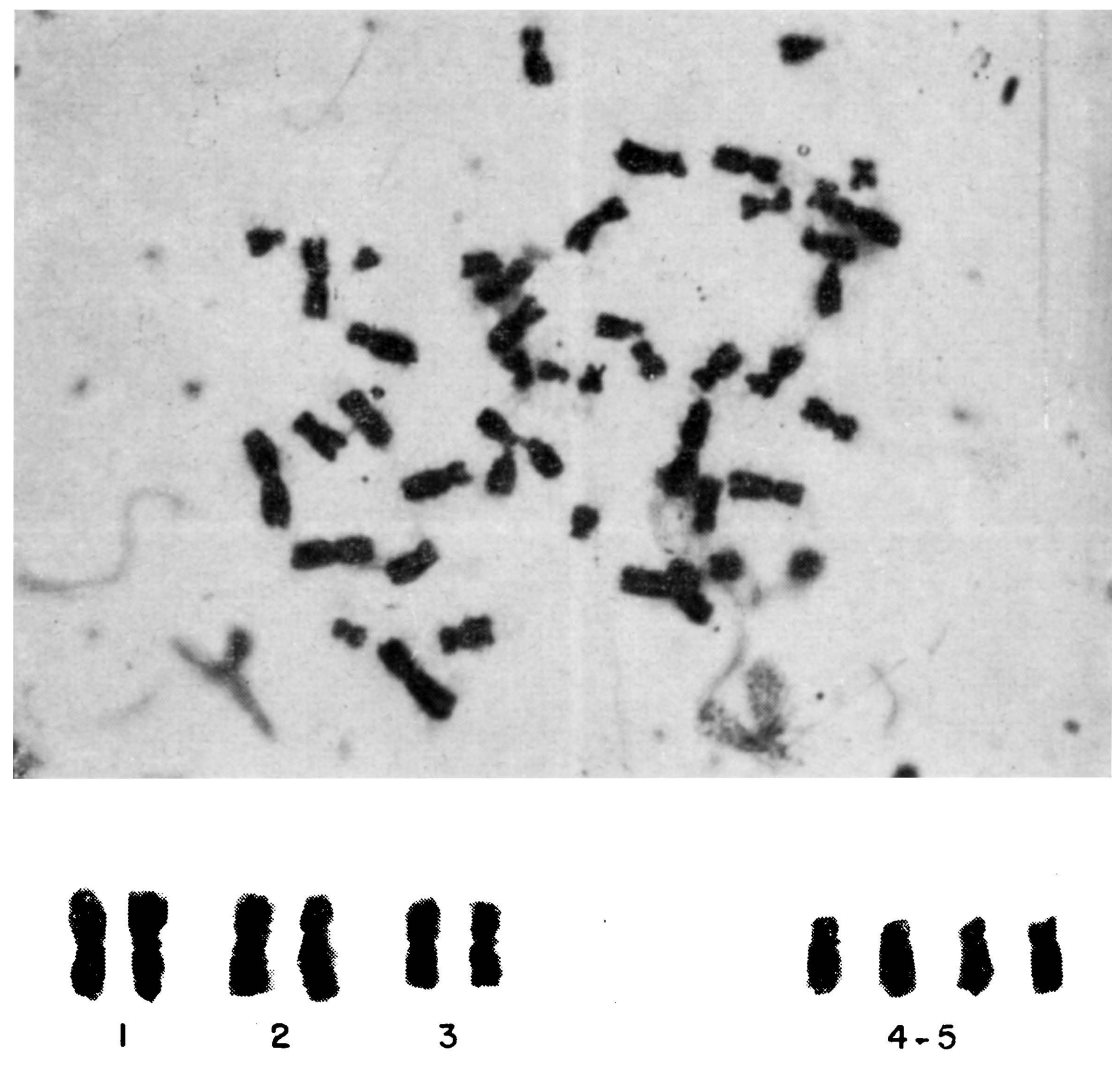

\section{1 $6-12$ e $x$}

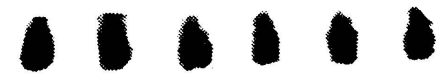

$3-15$

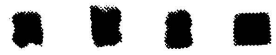

$19-20$

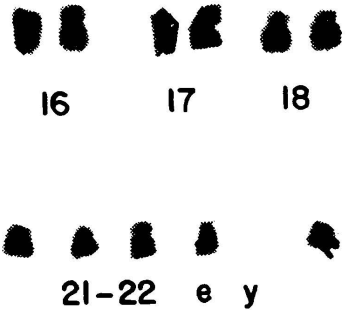

Fig. 2 - Caso E.F.C. Aspecto metafásico de uma das células examinadas e pareamento dos cromossomos classificados segundo a nomenclatura de Denver. $O$ número $e$ a configuração dos cromossomos são normais. 


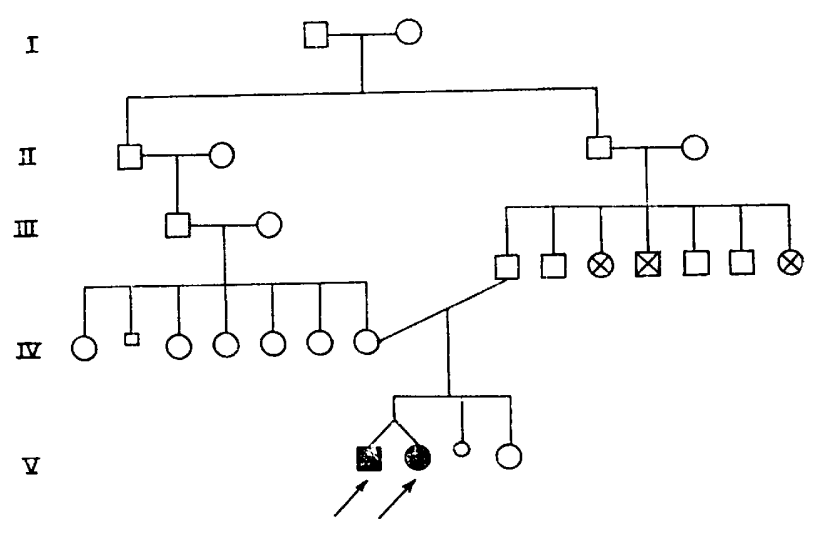

PACIENTES COM CRANIOSTENOSE

$\otimes \otimes$ SURDO-MUDOS

- O NATIMORTOS

Fig. 3 - Carta genealójica de nossos doentes, notando-se serem êles os únicos afetados com a craniostenose em tôda a genealogia

mica. Na realidade os pacientes são gêmeos dizigóticos (sexo diferente), o que afasta a possibilidade de que a craniostenose seja por mutação dominante, fenocópias ou influência de fatôres ambientais. Por outro lado, o exame cariotípico de um dos gêmeos (E.F.C.) não revelou aberrações cromossômicas, confirmando, portanto, um mecanismo gênico na determinação hereditária da craniostenose.

Embora o número de casos estudados seja muito pequeno, e insuficiente portanto para conclusões definitivas, nossos achados indicam que a obliteração precoce da sutura coronária e da sutura sagital são transmitidas hereditàriamente por gens recessivos raros de natureza autossômica. Além disso, mostram não haver especificidade quanto a determinação da sutura comprometida, pois, em nossos pacientes, a cbliteração afetava de maneira diversa a sutura sagital, enquanto que a sutura coronária estava obliterada apenas na menina.

\section{RESUMO}

É relatada a ocorrência de formas clínicas diversas de craniostenose em gêmeos de sexo diferente. A menina apresentava obliteração completa da sutura coronária e dos dois têrços anteriores da sutura sagital; no menino a sutura sagital era a única afetada. O estudo genético mostrou que a craniostenose independe de aberrações cromossômicas, indicando ser transmitida por gens recessivos raros de natureza autossômica. 


\section{SUMMARY}

Craniostenosis in twins: a genetic study.

The occurrence of two clinical forms of craniostenosis in twins of different sex born to consanguineous parents is reported. The girl showed total fusion of the coronary suture; in the boy sagittal suture was the only one involved. The genetic study showed that the craniostenosis was not associated with chromosomal anomalies and was related to rare autosomic recessive gens.

\section{REFERENCIAS}

1. ALMEIDA, G. M. \& BARROS, N. G. - Craniostenose: tratamento cirürgico. Considerações a respeito de 25 casos. Arq. Neuropsiquiat. (São Paulo) 23: 231-252, 1965.

2. BELL, H. S.; CLARE, F. B. \& WENTWORTH, A. F. - Familial scaphocephaly. J. Neurosurg. 18:239-241, 1961

3. BOFFANO, M. - Contributo casuistico allo studio della craniosinostosi precoce. Ann. Radiol. diagn. (Bologna) 35:30-55, 1962.

4. DEGENHARDT, K. H. - Malformaciones de la Cabeza y Columna Vertebral. In Becker, P. E.: Genética Humana, tradução espanhola. Toray, Barcelona, 1966, vol. II, pp. 507-614.

5. FAIRMAN, D. \& HORRAX, G. - Classification of craniostenosis. J. Neurosurg. 6:307-313, 1949.

6. FORD, F. R. - Prenatal Diseases and Developmental Defects of the Nervous System. In Ford, F. R.: Diseases of the Nervous System in Infancy, Childhhood and Adolescence. Charles Thomas, Springfield, 1960, pp. 158-302.

7. INGRAHAM, F. D.; ALEXANDER, J. F. \& MATSON, D. D. - Clinical studies in craniosynostosis. Analysis of fifty cases and description of method of surgical treatment. Surgery 24:518-541, 1948.

8. INGRAHAM, F. D. \& MATSON, D. D. - Craniostenosis. In Ingraham, F. D. \& Matson, D. D.: Neurosurgery of Infancy and Childhood. Charles Thomas, Springfield, 1954, pp. 83-104.

9. REILLY, B. J. - Craniosynostosis in the rachitic spectrum. J. Pediat. 64:396405, 1964.

10. SALdANHA, P. H. - Genética e Epidemiologia. In Farina, R.: Plástica de Nariz: Rinoplastias e Rinoneoplastias. Tipografia "O Calvário", São Paulo, 1966. pp. 51-104. 